\title{
THE RENORMALIZATION FLOW IN THE ADJOINT SU(2) LATTICE HIGGS MODEL ${ }^{1}$
}

\author{
R. BAIER ${ }^{2}$, C.B. LANG ${ }^{3}$ and H.-J. REUSCH ${ }^{2}$ \\ ${ }^{2}$ Fakultät für Physik, Universität Bielefeld, D-4800 Bielefeld, F.R. Germany \\ and \\ ${ }^{3}$ Institut für Theoretische Physik, Universität Graz, A-8010 Graz, Austria
}

Received 11 July 1988

\begin{abstract}
Applying the Monte Carlo renormalization group method we investigate the flow of coupling constants for the $8^{4}$ and $16^{4} \mathrm{SU}(2)$ lattice Higgs model with triplet Higgs fields. The couplings of the renormalized actions are determined using Schwinger-Dyson equations. From the flow we find new evidence for the existence of a tricritical point at finite values of the inverse gauge coupling $\beta$ but no indication for a discontinuity fixed point.
\end{abstract}

\section{Introduction}

The lattice version of the Georgi-Glashow model [1], which may be considered as a prototype model for the Higgs mechanism for grand unification, is of particular interest within the group of lattice Higgs models [2]. It shows a two-phase structure with separated phases: the confinement phase and the Higgs phase. It may further serve as a model that interpolates between the $O(3)$ scalar field theory, the pure $\mathrm{SU}(2)$ and $\mathrm{U}(1)$ gauge theories, respectively.

Although the structure of the phase diagram for this lattice model is reasonably well known [3-7], it is an open question where possible continuum limits may be taken. One obvious candidate is the critical point at vanishing gauge coupling, presumably in the universality class of the gaussian model. However, from the recent studies of the fundamental Higgs model [8-10] one expects that one can define there an effective, interacting field theory only at finite cut-off, i.e. at non-vanishing lattice spacing. If, however, there is another non-trivial fixed point (FP) in the model, it may exhibit different properties possibly related to a non-trivial continuum theory.

The situation is fascinating for the adjoint Higgs model: for the limiting case of the U(1) gauge model with the Wilson action data on bulk quantities support the first order nature of the phase transition (PT) [11]. The PT for the other limit, i.e.

\footnotetext{
${ }^{1}$ Supported in part by Deutsche Forschungsgemeinschaft, and by Fonds zur Förderung der Wissenschaftlichen Forschung in Österreich, Projekt P5965.
} 
for the $O(3)$ model, is second order and in the universality class of the gaussian model. It appears extremely hard numerically to decide on the basis of such bulk measurements, whether the order of the PT changes along the interpolating phase boundary [4]. Renormalization group methods may help in deciding whether there is a tricritical point (TCP) or not.

There are two established Monte Carlo (MC) methods to study lattice systems and their critical behaviour. One is by direct determination of the long distance properties through measurements of extended observables like correlation functions with the continuing struggle with boundary and other finite size effects. The other approach is by studying the renormalization properties of the system with regard to local observables defined on small length scales. This is the essence of the Monte Carlo renormalization group (MCRG) method introduced originally for spin systems [12].

Real space renormalization group (RG) transformations do not change the long distance behaviour of the system. They do change the scale of the lattice spacing, however, and the lattice action corresponding to the new set of block configurations in general will have differing coupling constants. Under repeated transformations one expects a FP behaviour of the block action. If the FP action contains essentially local interaction terms this approach allows a study of the FP properties based on local observables only.

Finite lattices pose a conceptual problem to the RG formalism, as well as a technical one. Firstly, the actions on lattices of different size live in different spaces and, strictly speaking, cannot be compared [13]. However, in practical calculations the comparison appears to be justifiable and has produced a lot of trustworthy and precise results [12], also for models with tricritical behaviour [14]. Secondly, especially in higher dimensional applications the actual lattice size is not big and may not allow for more than one or two blocking steps. Without sufficient luck (in this case a conspiracy between the simple form of the action and a favourable form of the block spin transformation (BST)) one will hardly observe a saturation of the RG flow in the space of couplings. Only seldom is the FP close enough to the specific form of the simulated action to allow convincing evidence. In most cases one has to rely on the overall trend of the flowlines exhibited in a calculation.

Three different kinds of FPs may be distinguished: trivial ones with vanishing correlation length, critical FPs with infinite correlation length, and the discontinuity fixed point (DFP) characteristic for transitions of first order [15]. Recently there arose a debate on the concept of DFPs and in some field theoretic models doubts on the analyticity of BSTs were expressed [16]. To the authors knowledge up to now no DFP has been observed within the context of gauge theories. On the other hand, in U(1) gauge theories [17-22] there is evidence for a hybrid type of FP, positioned on the first order part of the PT surface, with a flow away from the singular surface on the hot side and a flow towards a FP within the surface on the cold side. Similar discontinuous flows are observed in the four-dimensional $Z(2)$ gauge theory [23]. 
Concerning Higgs models we are confronted with the following situation: firstly, it is generally believed that the $\Phi^{4}$ model in four dimensions has only the gaussian FP and thus lies in the universality class of the non-interacting model. Secondly, based on renormalization group investigations it is argued that adding gauge interactions does not change this situation in the case of the fundamental Higgs model [9], although the existence of another additional FP is not definitely ruled out.

With regard to the model considered here there is the interesting possibility that the first order PT may change continuously into a second order one at finite value of $\beta$ and finite gauge-Higgs coupling $\kappa$. In this case there should appear a tricritical manifold as boundary between the first order and the second order critical manifolds, i.e. a point in the $(\beta, \kappa)$ plane. We may have two situations.

(i) The PT is first order all the way up the gaussian endpoint; then there may be a DFP somewhere on the phase boundary or a hybrid type of FP as discussed in the context of the pure U(1) gauge theory.

(ii) There is a tricritical point at finite values of $\beta$; then on the first order part of the phase boundary there may be a FP as before. There would be a FP somewhere in the tricritical manifold and if one succeeds to start exactly at the TCP in the $(\beta, \kappa)$ plane the flow should lead to this FP. In addition there is a critical FP which defines the universality class of the model situated along the critical part of the phase boundary; this FP may be gaussian.

In our analysis of the adjoint Higgs model we study the flow of observables and renormalized couplings under real space renormalization group transformations. Preliminary results have been presented elsewhere [24]. Here we discuss the final results for the phase structure: we find evidence for the existence of a TCP around $\beta=2.7, \kappa=0.65$. On the discontinuous part of the PT we find no evidence for a DFP with critical exponent $\nu=\frac{1}{4}$. In sect. 2 we present the notation and details of the methods, in sect. 3 the results on the flow in coupling- and operator space. In sect. 4 we discuss the eigenvalues of the linearized BST and in the final sect. 5 we summarize adding a few remarks on future explorations.

\section{Notation and formalism}

The gauge field is represented by the link variables $U_{x, \mu}$. Using Pauli matrices the adjoint Higgs field is represented by $\Phi_{x}=\mathrm{i} \phi_{x} \cdot \sigma$ where $\phi_{x}$ is a three-component unit vector $\left|\phi_{x}\right|=1$. The lattice action is

$$
\begin{aligned}
S= & \beta \sum_{x, \mu>\nu>0} \frac{1}{2} \operatorname{Tr} U_{x, \mu \nu}+\beta_{A} \sum_{x, \mu>\nu>0} \frac{1}{3} \operatorname{Tr}^{2} U_{x, \mu \nu} \\
& +\kappa \sum_{x, \mu>0} \frac{1}{2} \operatorname{Tr}\left(\Phi_{x} U_{x, \mu} \Phi_{x+\mu}^{\dagger} U_{x, \mu}^{\dagger}\right)
\end{aligned}
$$


and $U_{x, \mu \nu}$ denotes the usual plaquette variable [25]. This form may be considered as the fixed length (quartic Higgs coupling $\lambda \rightarrow \infty$ ) limit of the corresponding model with dynamical length. The phase structure and the topological properties of the fixed length model carry over to the situation with finite $\lambda$.

Most of our simulations are done on lattices of size $8^{4}$; at a few points in the phase diagram we also work on $16^{4}$ lattices. A three-hit Metropolis algorithm is used for the updating and we typically discard the first 20000 iterations. On the original lattice we choose a specific gauge where $\phi_{x}=(0,0,1)$. In the analysis BSTs and measurements are done every fifth update sweep. For given couplings up to 30000 configurations (out of 150000 updated ones) are analysed for lattices of size $8^{4}$. On the larger $16^{4}$ lattice the achieved statistics is much smaller: only $1000-2000$ iterations are discarded and from $6000 \mathrm{MC}$ iterations data are taken on every configuration. Error estimates are obtained by combining the measurements into several blocks [26].

\subsection{BLOCK SPIN TRANSFORMATION}

RG transformations introduce a change of the lattice spacing, the intrinsic cut-off. They should be local transformations of the field variables and are supposed to leave the long distance correlations of the ensemble of configurations invariant. In our MC calculation the configurations are determined in real space and therefore the BSTs should act on the field variables in real space, too. Gauge invariance has to be respected. We want to avoid gauge fixing - this is not necessary but convenient. Global Z(2) symmetry is unbroken; therefore the BST's do not have to respect it as long as an implicit global transformation is independent of the specific configuration (i.e. all block configuration variables may be multiplied simultaneously with elements of the center group $Z(2)$ ). Finally we want to stay within the given representation of the gauge group $S U(2)$; this requires certain non-linearities of the BSTs.

We work with BSTs with a scale factor of 2 and the block lattice is imbedded in the original one. For gauge field link variables the block link between the block lattice sites $x^{\prime}$ and $x^{\prime}+\mu$ may be constructed from sums over paths connecting the sites on the original lattice. Generalizing the transformation in [27] we obtain the block link $U_{x^{\prime}, \mu}^{\prime}$ from the normalized sum over gauge link products along three types of paths,

$$
\begin{aligned}
W_{x^{\prime}, \mu}(U)= & \rho_{0} U_{x, \mu} U_{x+\mu, \mu}+\rho_{1} \sum_{\nu \perp \mu} U_{x, \nu} U_{x+\nu, \mu} U_{x+\nu+\mu, \mu} U_{x+2 \mu, \nu}^{\dagger} \\
& +\rho_{2} \sum_{\nu \perp \mu}\left(U_{x, \nu} U_{x+\nu, \mu} U_{x+\mu, \nu}^{\dagger} U_{x+\mu, \mu}+U_{x, \mu} U_{x+\mu, \nu} U_{x+\nu+\mu, \mu} U_{x+2 \mu, \nu}^{\dagger}\right) \\
U_{x^{\prime}, \mu}^{\prime}(U)= & \frac{W_{x^{\prime}, \mu}(U)}{\sqrt{\operatorname{det}\left(W_{x^{\prime}, \mu}(U)\right)}} .
\end{aligned}
$$




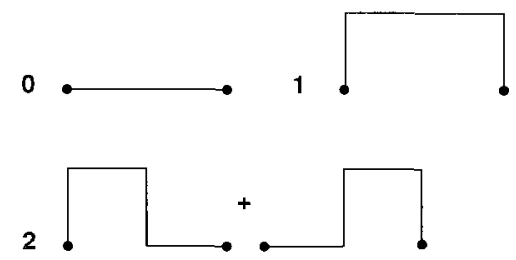

Fig. 1. The paths considered for the gauge field BST, the numbers correspond to the notation of eq. (2.2).

The shapes of the paths are shown in fig. 1 . The parameters $\rho_{i}$ determine the relative weights of the path shapes; $\rho_{0}$ may be set to 1 without loss of generality.

In general any BST obeying the above conditions should work; however, the positions of FPs in parameter space and hence the renormalization flow may differ depending on the choice of transformation. Within SU(2) gauge theories there have recently been studies in this respect [28,29]. In ref. [29] the factors in eq. (2.2) are optimized for the pure SU(2) gauge theory in order to minimize the RG flow away from the single parameter Wilson action. It turns out that it is possible to improve the flow behaviour, i.e. to move the renormalized trajectory (RT) substantially closer to the Wilson axis. However, there is still a clear RG flow towards other gauge couplings, even for the "optimal" choice. This is our main reason to keep at least one more gauge coupling, the adjoint one, in the action eq. (2.1). For the parameters we use the values $\rho_{1}=-6.0, \rho_{2}=-0.2$ due to an earlier result of the authors of ref. [29] which is different from the final "optimized" values $\rho_{1}=-0.70$, $\rho_{2}=-0.18$ [29]. However, for our purpose the difference in the flow is not significant.

In our calculations we also determine the coupling flow in the $\left(\beta, \beta_{A}\right)$ plane for the pure SU(2) gauge theory. Starting with configurations obtained for the pure Wilson action $\left(\beta_{A}=0\right)$ one observes a misleading effect. At small values of $\beta$ the flow leads towards smaller values of this coupling as expected. Above a certain $\beta$ of the order of $\approx 2.7$ the flow apparently leads towards larger values of $\beta$. Although superficially this seems to indicate a FP at $\beta \simeq 2.7$ a closer inspection of the flow in the $\left(\beta, \beta_{A}\right)$ plane shows that in both cases the flow runs toward negative $\beta_{A}$ and eventually, after sufficiently many RG steps, turns and continues to follow a common RT towards the trivial FP at vanishing $\beta$ and $\beta_{A}$. This confirms the results of a previous study [30], in which a different method for the determination of the renormalized couplings is applied. There it is also noted that this specific flow pattern may be caused by the nearby phase transition line present in the $\left(\beta, \beta_{A}\right)$ plane [31]. Obviously this effect is overlooked in the analysis of ref. [10], although the same BST, eq. (2.2), with $\rho_{i}=(1,1,1)$ is used: for the blocking $6^{4} \rightarrow 3^{4}$ a flow towards the trivial FP is indicated even for $\beta$ as large as $\beta \simeq 5$. This detailed 
discussion should point to possible difficulties in the interpretation of coupling flows, since a similar observation may also be relevant for the full Higgs system.

The Higgs field variables are blocked in analogy to the transformation popular in scalar field theory. The block variables $\Phi_{x^{\prime}}^{\prime}$ are obtained from the sum over all Higgs field variables $\Phi_{y}$ within a $2^{4}$ hypercube $\mathrm{H}_{x}$. The lower left site $x$ of the hypercube is identified with the site $x^{\prime}$ on the block lattice. The Higgs variables are gauge transformed along the shortest paths $P_{x, y}$ connected to this site. In case of multiple paths the arithmetic mean over these contributions is considered. We define the normalized block variables by

$$
\begin{aligned}
\chi_{x^{\prime}} & =\sum_{y \in \mathrm{H}_{x}} \frac{1}{n_{y}} \sum_{P_{x, y}} \Pi_{x, y} \Phi_{y} \Pi_{x, y}^{\dagger}, \\
\Phi_{x^{\prime}}^{\prime} & =\frac{\chi_{x^{\prime}}}{\sqrt{\operatorname{det}\left(\chi_{x^{\prime}}\right)}},
\end{aligned}
$$

where $n_{y}$ denotes the multiplicity of shortest paths, and $\Pi_{x, y}$ the ordered product of link variables along this path. No attempts to improve the $R G$ flow are tried here.

\subsection{DETERMINATION OF BLOCK COUPLINGS}

Given an ensemble of configurations, what are the coupling constants of the corresponding action? A variety of methods has been proposed to determine the renormalized couplings [32-34]. Most of the successful methods are based on measurements on the blocked configurations only and do not involve comparisons with statistically independently generated test ensembles. Swendsen's method [33] is well suited for the determination of a larger number of couplings but is numerically quite demanding, especially for the situation of non-abelian gauge theories. The method based on Schwinger-Dyson equations [34] is easier to handle and comparatively faster if one wants to determine only a few couplings.

We determine only three couplings: $\beta, \beta_{A}$ and $\kappa$. The corresponding SchwingerDyson equations are summarized in the appendix. As may be seen this set of equations is overcomplete. One either uses some of the equations only as a consistency check or one chooses to minimize a mean square deviation from zero of the four equations, weighted by the statistical error involved in the relevant terms in each equation. We decide for the second alternative which provides a measure of the reliability of the determined coupling values in terms of the achieved $\chi^{2}$.

We perform various tests on the reliability of this method, one of them reproducing the three couplings for ensembles of configurations generated for various non-vanishing values of these three couplings. The correct values are reproduced at least within three digits accuracy.

In order to get an estimate of finite size effects we compare the results obtained for two blocking steps from runs blocking $8^{4} \rightarrow 4^{4} \rightarrow 2^{4}$ with runs blocking from 
TABLE 1

Comparison of the flow in the couplings $\beta, \kappa, \beta_{A}$ after the first and second blocking step for two lattice sizes $8^{4}$ and $16^{4}$, respectively

\begin{tabular}{|c|c|c|c|c|c|c|}
\hline \multirow{2}{*}{$\begin{array}{l}\text { Block } \\
\text { step }\end{array}$} & \multicolumn{3}{|c|}{$8 * * 4$} & \multicolumn{3}{|c|}{$16 * * 4$} \\
\hline & $\beta$ & $\kappa$ & $\beta_{A}$ & $\beta$ & $\kappa$ & $\beta_{A}$ \\
\hline input & 1.8400 & 1.5236 & 0.0000 & 1.8400 & 1.5236 & 0.0000 \\
\hline 0 & $\begin{array}{c}1.8398 \\
(0.0007)\end{array}$ & $\begin{array}{c}1.5236 \\
(0.0006)\end{array}$ & $\begin{array}{c}0.0000 \\
(0.0005)\end{array}$ & $\begin{array}{c}1.8396 \\
(0.0003)\end{array}$ & $\begin{array}{c}1.5233 \\
(0.0003)\end{array}$ & $\begin{array}{l}-.0001 \\
(0.0003)\end{array}$ \\
\hline 1 & $\begin{array}{c}1.6344 \\
(0.0032)\end{array}$ & $\begin{array}{c}1.4199 \\
(0.0023)\end{array}$ & $\begin{array}{l}-.0254 \\
(0.0024)\end{array}$ & $\begin{array}{c}1.6316 \\
(0.0008)\end{array}$ & $\begin{array}{c}1.4193 \\
(0.0009)\end{array}$ & $\begin{array}{l}-.0260 \\
(0.0006)\end{array}$ \\
\hline 2 & $\begin{array}{c}1.6971 \\
(0.0146)\end{array}$ & $\begin{array}{c}1.4254 \\
(0.0106)\end{array}$ & $\begin{array}{c}0.0247 \\
(0.0099)\end{array}$ & $\begin{array}{c}1.7448 \\
(0.0037)\end{array}$ & $\begin{array}{c}1.4305 \\
(0.0026)\end{array}$ & $\begin{array}{l}-.0372 \\
(0.0024)\end{array}$ \\
\hline input & 2.4000 & 0.9500 & 0.0000 & 2.4000 & 0.9500 & 0.0000 \\
\hline 0 & $\begin{array}{c}2.3992 \\
(0.0006)\end{array}$ & $\begin{array}{c}0.9501 \\
(0.0002)\end{array}$ & $\begin{array}{c}0.0006 \\
(0.0004)\end{array}$ & $\begin{array}{c}2.3994 \\
(0.0003)\end{array}$ & $\begin{array}{c}0.9494 \\
(0.0002)\end{array}$ & $\begin{array}{c}0.0001 \\
(0.0002)\end{array}$ \\
\hline 1 & $\begin{array}{c}2.4847 \\
(0.0012)\end{array}$ & $\begin{array}{c}1.1957 \\
(0.0026)\end{array}$ & $\begin{array}{l}-.0857 \\
(0.0016)\end{array}$ & $\begin{array}{c}2.4838 \\
(0.0015)\end{array}$ & $\begin{array}{c}1.1929 \\
(0.0008)\end{array}$ & $\begin{array}{l}-.0859 \\
(0.0009)\end{array}$ \\
\hline 2 & $\begin{array}{c}2.8995 \\
(0.0163)\end{array}$ & $\begin{array}{c}1.5718 \\
(0.0027)\end{array}$ & $\begin{array}{c}-.0329 \\
(0.0101)\end{array}$ & $\begin{array}{c}2.9572 \\
(0.0100)\end{array}$ & $\begin{array}{c}1.5734 \\
(0.0027)\end{array}$ & $\begin{array}{l}-.1172 \\
(0.0056)\end{array}$ \\
\hline
\end{tabular}

The input couplings are well reproduced by the Schwinger-Dyson method (cf. block step 0). Estimates of the statistical errors are given in parentheses.

$16^{4} \rightarrow 8^{4} \rightarrow 4^{4}$. The couplings determined after the first blocking step are in excellent agreement. After the second blocking step the flow with respect to $\beta$ and $\kappa$ remains consistent. Two typical examples are shown in table 1 for starting configurations in the cold phase.

\subsection{OPERATORS AND LINEARIZED BST}

Because we determine only the three renormalized couplings: $\beta, \beta_{A}$, $\kappa$, the observed flow constitutes a projection from the, essentially infinite dimensional, space of all possible couplings into this three-parameter subspace. As concerns the flow in the space of further gauge-couplings we try to minimize it by a suitable choice of the BST (cf. the discussion following eq. (2.2)). However, we cannot exclude that the actual flow appears obscured due to this projection and we try to be very careful in the final interpretation.

The measured observables on the blocked lattices provide another source of information on the flow, now due to direct measurements without truncating the 


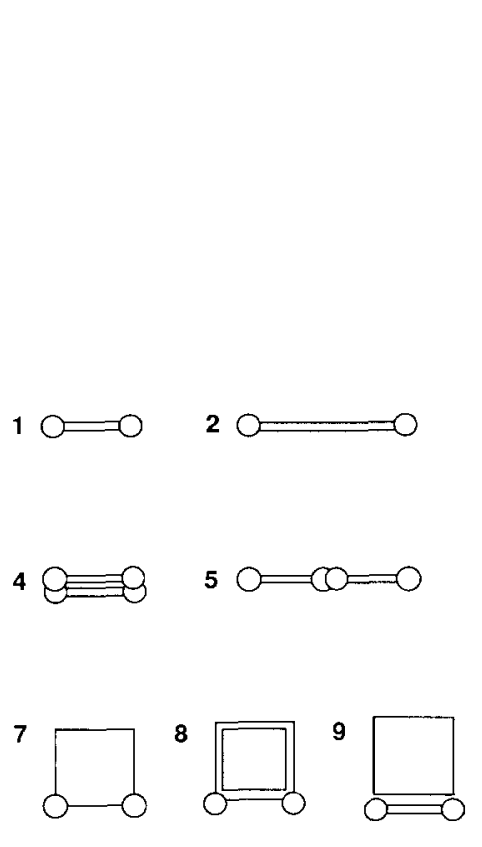

(a)
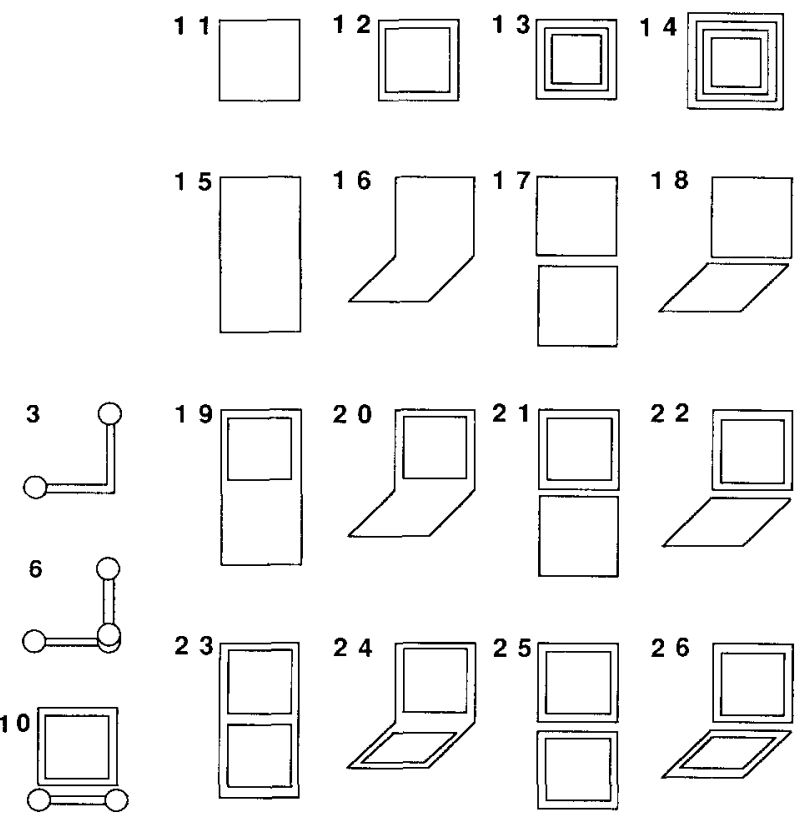

(b)

Fig. 2. The observables measured on the original and the blocked configurations in a symbolic notation. Each term is built from products of SU(2) traces over the various objects. A closed loop of links denotes a product of the corresponding gauge fields; Higgs fields are represented by circles at sites and, due to their representation, must have one incoming and one outgoing link. Operators 1,11 and 12 correspond to the three terms in the lattice action. Fig. $2 a$ lists operators involving Higgs variables, fig. $2 b$ pure gauge

field operators.

number of couplings. Here we consider a substantially larger set of operators, i.e. directions in the corresponding space of observables: we take the 26 operators which enter the Schwinger-Dyson equations. This set includes the plaquette and other Wilson loops in fundamental and higher representations, non-nearest neighbour Higgs-Higgs coupling terms, and products of Higgs fields connected by gauge transformations along various paths. Fig. 2 depicts the various terms symbolically.

We identify the set of values obtained for these observables for an ensemble of configurations with a point in the multidimensional operator space. PTs and other structures in the coupling space have their equivalent structure in this operator space. The RG flow structure can be studied independently based on these results and this provides a consistency check on the structure obtained from the coupling flow.

At a FP the BST in coupling space may be linearized defining the matrix

$$
T_{\alpha \beta}^{(n+1, n)}=\frac{\partial K_{\alpha}^{(n+1)}}{\partial K_{\beta}^{(n)}},
$$


where $K_{\alpha}^{(n)}$ denotes the coupling $K_{\alpha}$ for the ensembles of configurations after $n$ blocking steps. The number of eigenvalues $\lambda>1$ of the $T$ matrix determines the number of relevant couplings.

At a DFP [15] there is only one such eigenvalue $\lambda=s^{d}$, where $s$ denotes the scale factor ( 2 in our case) and $d=4$, the number of dimensions.

At a critical FP the relevant eigenvalues are related to the critical indices $\nu_{i}$

$$
\lambda_{i}=s^{1 / v_{i}},
$$

where the largest eigenvalue gives the critical exponent for the correlation length. At a standard critical FP there is one relevant eigenvalue (e.g. $\lambda=4$ or $\nu=\frac{1}{2}$ at a gaussian FP). At a tricritical FP one expects in general two relevant eigenvalues, but it may happen, as e.g. in the $d=4 \Phi^{6}$ theory, that there is one relevant and one marginal $(\lambda=1)$ eigenvalue [22].

The $T$ matrix may be determined in an approximate way from correlations between operators measured after $n$ and $n+1$ blocking steps [12]. This determination should become more stable when one includes more operators in the analysis. The operators which affect the leading eigenvalues substantially will be important contributors to the renormalized action. The determination is reliable only close to FPs of the renormalization flow. The leading eigenvalues, however, behave in a smooth way even away from the FP.

\section{Results for the MCRG flow}

In the subsequent discussion we collect evidence from two groups of results. First, from the renormalized couplings, second from the measured operator expectation values and their cross-correlations. Fig. 3 shows the measured flow - the lines interpolate between the points on the $8^{4} \rightarrow 4^{4} \rightarrow 2^{4}$ lattices - in a two-parameter subspace of couplings (fig. 3a) and operators (fig. 3b), respectively. We observe the same structure of the flow pattern in both spaces. The results may be organized naturally by discussing two regions in the phase diagram of the starting lattice: (i) $\beta \leq 2.4$ and (ii) $\beta>2.4$.

\section{1. $\beta \leq 2.4$}

Here clear two state signals and metastable states are observed in the simulation (cf. ref. 4). As a further example we plot in fig. 4 the dependence of the mean plaquette versus $\beta$ when crossing the PT near $\beta \simeq 1.755$ : a clear gap is visible. A similar behaviour is observed for the other, e.g. link, operators.

Starting on the confinement branch at and below the PT we find a flow directly towards the trivial ("hot") FP at $\beta=0, \kappa=0$. The values of $\beta_{A}$ on the blocked lattices remain negligible. No flow to an attractive RT is identified in the confined phase. 


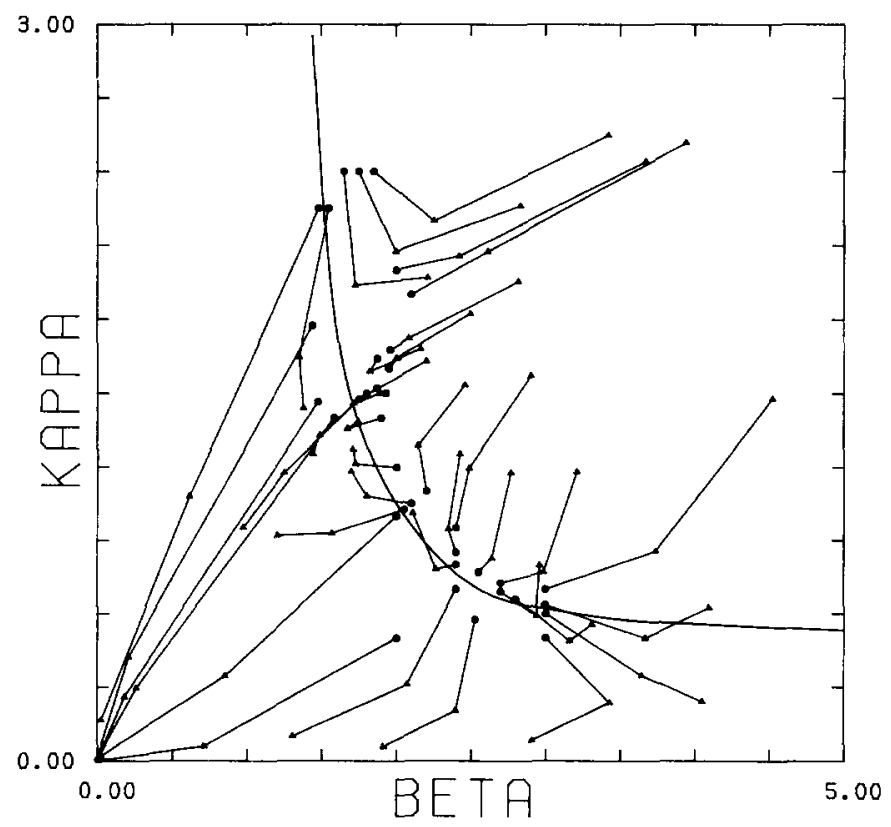

(a)

Fig. 3a. Flow of couplings $(\beta, \kappa)$ for two blocking steps in the neighbourhood of the PT (solid curve). The starting values are indicated by circles.

On the Higgs side the flow in general seems to be attracted by a trajectory moving toward large values of $\beta$ and $\kappa$. Close to the PT and to its intersection with the attractor we observe a behaviour peculiar at first sight: the flow crosses the PT and seems to move towards the "hot" FP. For these runs - even starting in the metastable phase - we make sure that no phase flips occur during the measurements. Eventually the speed of flow in this direction slows down and for some of the trajectories, which start not too close to the PT, we can still observe a turn after the second BST back towards the Higgs side of the phase diagram, following the direction of the attractive RT. Independent information from the measured block operators confirms this structure. A typical flow "crossing" the PT line in the $(\beta, \kappa)$ plane is illustrated in table 2a: it starts in the Higgs phase, flows to smaller couplings and then turns back during the $8^{4} \rightarrow 4^{4} \rightarrow 2^{4}$ blocking. Next we take the values for $\beta, \kappa, \beta_{A}$ after the first blocking step again as input values on the $8^{4}$ lattice and perform two blocking steps. The resulting flow now quickly tends to the trivial FP (table 2b), in contrast to the previous flow pattern (table 2a).

We come to the conclusion that the flow stays in or close to the PT manifold but also runs into directions in coupling space that have not been determined (i.e. neither one of $\left.\beta, \beta_{A}, \kappa\right)$. The crossing of the PT line is just a projection effect. 


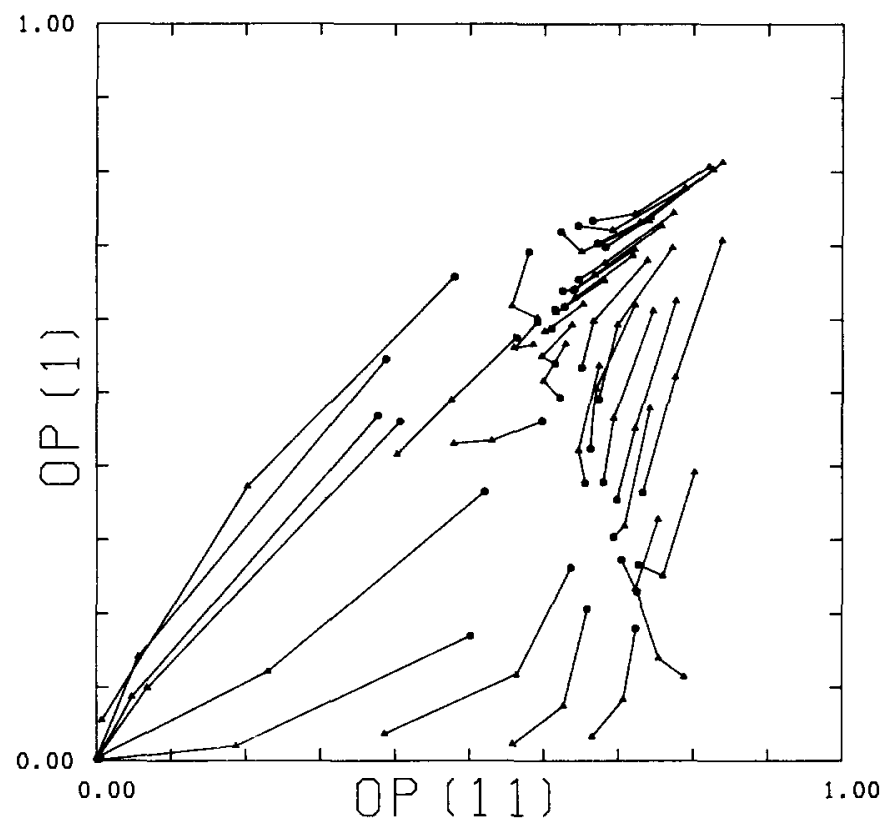

(b)

Fig. 3b. Flow of operators: plaquette ( $\equiv$ operator 11 of fig. 2) versus link ( $\equiv$ operator 1 of fig. 2 ) in the neighbourhood of the PT.

Following the actual flow over more than two blocking steps is almost impossible in the limited coupling space (on lattices as large as $16^{4}$ ), since many more couplings are to be determined for a successful restart in the same phase.

In case there is a FP in the PT manifold, then it is reached only from the Higgs side of the PT and it may be quite far away from the three-coupling manifold. From the analysis of the linearized BST (see sect. 4) we find that e.g. operator 7 provides important contributions and is therefore a candidate for non-negligible further interaction couplings.

This behaviour appears very similar to the one in the $U(1)$ lattice gauge theory [18-22], (cf. the discussion in sect. 1). As suggested by Hasenfratz et al. [16,21] it might be associated with a singular behaviour of the BST (first observed for the three-state Potts model [35]). In U(1) the Coulomb phase - like the Higgs phase in the adjoint Higgs model - has a massless photon. Therefore in both theories the FPs, which do not appear to be of the DFP type expected at first order PTs [15], may have the same hybrid nature. An interpretation advocated in refs. $[19,20,36]$ holds responsible the occurrence of monopole loops, winding around the lattice, as due to periodic boundary conditions. MC simulations may not give justice to these non-local objects and thus may considerably delay equilibration from the cold side. 
R. Baier et al. / Lattice Higgs model

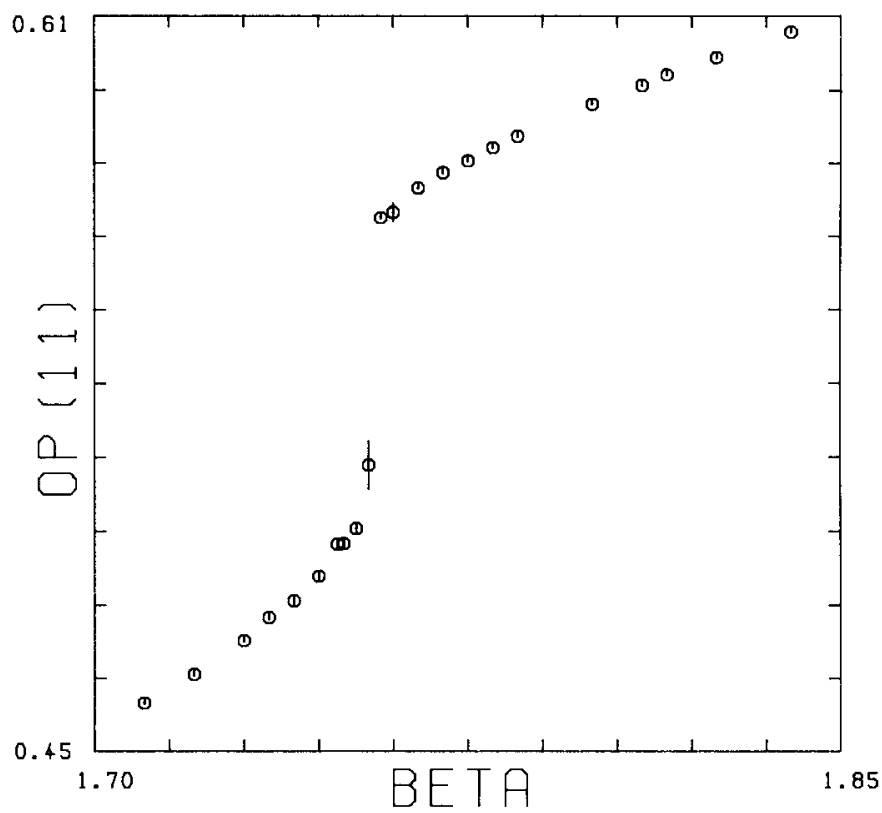

Fig. 4. Mean plaquette versus $\beta$ from starting configurations along a line $\kappa=0.36075+0.632 \beta$ crossing the discontinuous PT.

TABLE 2a

Example for a coupling flow "crossing" the PT line in the $(\beta, \kappa)$ plane and turning back

\begin{tabular}{clcc}
\hline Size & \multicolumn{1}{c}{$\beta$} & \multicolumn{1}{c}{$\kappa$} & $\beta_{A}$ \\
\hline $8^{4}$ & 1.9000 & 1.4000 & 0.0000 \\
$4^{4}$ & $1.6732(17)$ & $1.3571(9)$ & $-0.0274(11)$ \\
$2^{4}$ & $1.7444(55)$ & $1.3858(41)$ & $-0.0007(43)$ \\
\hline
\end{tabular}

TABLE $2 b$

Starting with the $4^{4}$ couplings of table 2 a, however, the flow tends quickly towards the trivial FP

\begin{tabular}{cllc}
\hline Size & \multicolumn{1}{c}{$\beta$} & \multicolumn{1}{c}{$\kappa$} & $\beta_{A}$ \\
\hline $8^{4}$ & 1.6732 & 1.3571 & -0.0274 \\
$4^{4}$ & $0.3318(7)$ & $0.3220(8)$ & $0.0011(8)$ \\
$2^{4}$ & $0.0017(22)$ & $0.0101(21)$ & $0.0027(42)$ \\
\hline
\end{tabular}


Therefore it cannot be definitely excluded that the indicated FP on the Higgs side of the PT is a real critical FP.

On the other hand the flow in the region $\beta \leq 2.4$ starting in the Higgs phase may also be interpreted without assuming at all the existence of a FP on the PT manifold. Obscured by projection effects in fig. 3 it may be that - similar to the flow pattern in the confinement phase - the flow lines leave the PT surface and become attracted by a trivial FP (at infinite couplings) even without approaching a common RT.

\section{2. $\beta>2.4$}

In this region no uncontroversial two-state signal is observed in the bulk measurements [4], possibly, because of very long relaxation times. Although a very small gap is not excluded by the MC data, we are not able to demonstrate it either. Altogether these results are consistent with the possible existence of a second order PT.

To start with the conclusion of the MCRG study: there is evidence for the existence of a TCP in the $(\beta, \kappa)$ plane around $(2.7(1), 0.65(5))$ which acts repulsive to the observed flow (fig. 3). As mentioned already, an ordinary critial FP with one relevant parameter attracts the flow from points within the critical surface and repels only in the direction leading away from the surface. In contradistinction, a TCP has two relevant directions, one of them leading away from the PT surface, and another one staying in the surface, but leading away from the tricritical boundary manifold between the first and the second order part of the PT manifold. It is the latter situation which we deduce from the flow picture for the couplings and operators; the relevant directions appear to have non-vanishing components in the $(\beta, \kappa)$ plane.

Of course, from these data alone it cannot be excluded that there is a flow into other, unobserved directions in parameter and operator space. In the worst case the flow (fig. 3) - in analogy to the pure $\mathrm{SU}(2)$ gauge theory near $\beta=2.7$ as discussed in sect. 2.1 - may only mimic tricritical behaviour, but it is actually caused by a different nearby singularity. In sect. 4 we give, however, additional evidence for the TCP interpretation.

The positions of the TCP and of the corresponding FP are difficult to determine precisely. In order to obtain reasonable estimates we use the linear approximation eq. (2.4) and extrapolate

$$
K_{\alpha}^{*}=K_{\alpha}^{(0)}+\sum_{\beta=1}^{3}\left[\left(1-T^{(1,0)}\right)^{-1}\right]_{\alpha \beta}\left(K^{(1)}-K^{(0)}\right)_{\beta},
$$

starting with configurations near the presumed TCP in the $(\beta, \kappa)$ plane. In a first set 
TABLE 3

The values for the fixed point $\left(\kappa^{*}, \beta^{*}, \beta_{A}^{*}\right)$ estimated from eq. (3.1) for runs with $\beta=2.55$ crossing the PT at $\kappa_{\mathrm{PT}}=0.700(5)$ by varying $\kappa$

\begin{tabular}{cccccc}
\hline$\kappa$ & $\lambda_{1}$ & $\lambda_{2}$ & $\kappa^{*}$ & $\beta^{*}$ & $\beta_{A}^{*}$ \\
\hline 0.685 & 2.34 & 1.69 & 2.55 & 2.95 & -2.80 \\
& $(0.24)$ & $(0.23)$ & $(1.97)$ & $(0.26)$ & $(3.37)$ \\
0.695 & 2.23 & 1.44 & 0.94 & 2.82 & -.15 \\
& $(0.25)$ & $(0.16)$ & $(0.03)$ & $(0.17)$ & $(0.13)$ \\
0.705 & 3.01 & 1.82 & 0.84 & 2.67 & -.04 \\
& $(0.14)$ & $(0.05)$ & $(0.02)$ & $(0.06)$ & $(0.06)$ \\
0.715 & 3.75 & 1.54 & 0.79 & 2.77 & -.15 \\
& $(0.33)$ & $(0.07)$ & $(0.01)$ & $(0.04)$ & $(0.03)$ \\
0.725 & 3.18 & 1.74 & 0.78 & 2.67 & -.07 \\
& $(0.16)$ & $(0.11)$ & $(0.01)$ & $(0.06)$ & $(0.06)$ \\
0.745 & 3.30 & 1.65 & 0.75 & 2.69 & -.10 \\
& $(0.02)$ & $(0.01)$ & $(0.01)$ & $(0.03)$ & $(0.02)$ \\
\hline
\end{tabular}

The two relevant eigenvalues are also given. Estimates of the statistical errors are given in parentheses.

of runs we start at $\beta=2.55$ and vary $\kappa$ across the PT, where $\kappa_{\mathrm{PT}}=0.700(5)$. It turns out that these couplings cross the discontinuous part of the PT line, i.e. left to the presumed TCP. The values for $\kappa^{*}, \beta^{*}, \beta_{A}^{*}$ obtained from the blocking step $8^{4} \rightarrow 4^{4}$, are summarized in table 3 . Above the Higgs phase we find rather stable values for $K_{\alpha}^{*}$ with the average $\beta^{*}=2.70(5), \kappa^{*}=0.79(3), \beta_{A}^{*}=-0.09(5)$. Simulating the system with these values on the $8^{4}$ lattice the iterated result becomes $\beta^{*}=2.69(2)$, $\kappa^{*}=0.715(60), \beta_{A}^{*}=-0.11(2)$, again very close to the previous values. We also check that after further restarts the evaluated $K_{a}^{*}$ 's remain consistent with this preferred point, although we do not succeed in localizing the FP with higher precision. We conclude that the given couplings provide a good estimate for the position of the tricritical FP.

When starting at larger values of $\beta$, i.e. at $\beta=3.0$, near the continuous PT we find $\beta^{*} \leq 2.9$, however, with errors too large for further sensible iterations with the help of eq. (3.1); these starting configurations are obviously not close enough to the FP estimated before. From this procedure we also derive the estimate for the TCP in the $(\beta, \kappa)$ plane, given above, by taking into account the dependence of $\kappa_{\mathrm{PT}}(\beta)$ on the PT for $\beta_{A}=0$.

At larger $\beta, \beta>3.0$, the flow runs towards still larger values of the gauge coupling, turning away from the PT line. This indicates that the gaussian FP at $\beta=\infty$ is the attractor for the critical part of the PT. 


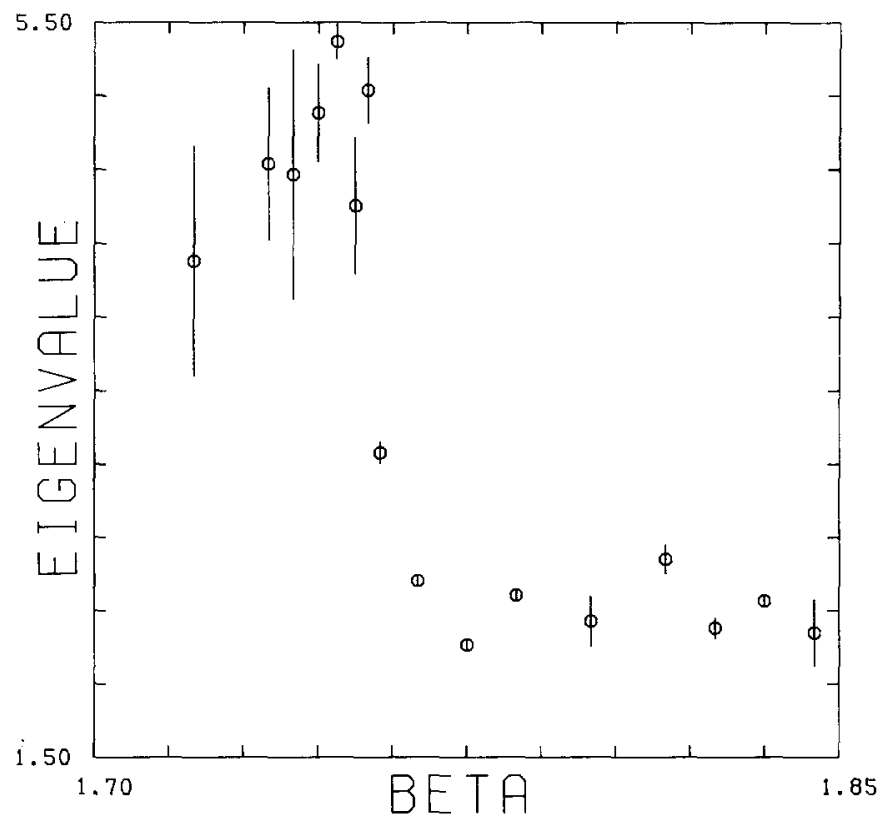

Fig. 5. The largest eigenvalue of the linearized BST $T^{(1,0)}$ (involving 5 operators) from starting configurations along the same line $\kappa(\beta)$ crossing the discontinuous PT as in fig. 4.

\section{Critical exponents}

In the region where one has a clear discontinuity signal (the region $\beta \leq 2.4$ as discussed in subsect. 3.1) the eigenvalues of the linearized BST should assume rather large values $\simeq 16$, if there is a DFP. We determine the eigenvalues of $T^{(1,0)}$ taking into account correlations between operators 1 (link), 11 (plaquette), 12 (adjoint plaquette), 2 and 7 (according to the notation in fig. 2). Fig. 5 exhibits the results for starting configurations along a line $\kappa=0.36075+0.632 \beta, \beta_{A}=0$. This line is chosen to represent - near the PT - the possible attractive RT indicated by the flow (fig. 3 ) in the cold phase. Only one eigenvalue is larger than 1.

Comparing this eigenvalue, obtained for operators $1,2,11,12$ only, with that for the complete set one finds an important contribution due to the operator 7 . This may indicate that there is a non-negligible renormalization flow into the direction of such a term in the action. It is somewhat surprising that operator 7 appears to be more important than, e.g. the linear next-to-nearest neighbour Higgs coupling operator 2.

The PT on the given line is at $\beta \simeq 1.755$. On the hot side the eigenvalue increases continuously towards the PT up to a maximum of $\lambda_{\max } \simeq 5.5(1.0)$ but it stays definitely below the DFP value 16 . On the Higgs side the value is of the order $\simeq 2.5$, slowly decreasing towards larger $\beta$. The overall behaviour resembles that 
TABLE 4

The two relevant eigenvalues of $T^{(n+1, n)}$ for $n=0,1$ (involving 5 operators) evaluated from the starting configuration $\beta=2.69, \kappa=0.715, \beta_{A}=-0.11$

\begin{tabular}{ccc}
\hline Block step & $\lambda_{1}$ & \multicolumn{1}{c}{$\lambda_{2}$} \\
\hline $8^{4} \rightarrow 4^{4}$ & $3.26(15)$ & $1.46(9)$ \\
$4^{4} \rightarrow 2^{4}$ & $3.25(62)$ & $1.61(12)$ \\
\hline
\end{tabular}

observed for the U(1) gauge theory [19]. It does not give the impression of a DFP but it may be indicative of a non-analytic behaviour at the PT. When comparing figs. 4 and 5 we observe that the gap in the mean plaquette and the discontinuous jump in the eigenvalue occur at the same value of $\beta$.

In the runs, already discussed in subsect. 3.2, close to the presumed TCP we always find two relevant eigenvalues (determined from the same five operators as above). Typical values are already included in table 3 for the $\beta=2.55$ runs: one may observe even a small jump in the $\lambda$ 's, i.e. $\Delta \lambda_{1} \simeq 0.8$, when crossing the discontinuous PT (cf. fig. 5). In contrast, for the corresponding runs at $\beta=3.0$ the eigenvalues are much smoother, slowly rising from smaller values on the confinement side to larger values $(<4)$ on the Higgs side. This gives further indication that indeed the order of the PT is changing between $\beta=2.55$ and $\beta=3.0$. The actual estimates for these two relevant eigenvalues are: the larger one assumes values between 3.0-3.8, the second largest between 1.5-1.8. Starting the BSTs at $\beta^{*}=2.69, \kappa^{*}=0.715$, $\beta_{A}^{*}=-0.11$, the preferred estimate for the FP, the corresponding eigenvalues are given in table 4: these eigenvalues show a very small variation with respect to the RG iterations.

Although we do not exactly know the systematic errors, e.g. due to truncations and finite size effects, our data indicate that the critical exponent $\nu$ is larger than $\frac{1}{2}$ and that the crossover exponent $\ln \lambda_{2} / \ln \lambda_{1}$ is different from zero.

\section{Conclusion and outlook}

Fig. 6 summarizes schematically the observed renormalization flow in the couplings. The flow in the $\beta_{A}$ direction is of little significance for the analysis, since in general $\beta_{A}$ remains very small after two BSTs. In the region $\beta \leq 2.4$ there is a clear signal for a discontinuous transition, but no indication of a DFP. On the confinement side the flow quickly leaves the PT and tends towards the "hot", trivial FP. On the Higgs side there is a signal of an attractive RT and of a flow within the PT surface, eventually moving away following this RT. In the region above $\beta>2.5$ we find strong indications for a TCP at the PT around $\beta \approx 2.7$ with two relevant directions.

There may be a relation between the position of the TCP with the structure of the PTs in the phase diagrams in the $\left(\beta, \beta_{A}\right)$ planes of the pure $\mathrm{SU}(2)$ and $\mathrm{U}(1)$ gauge 


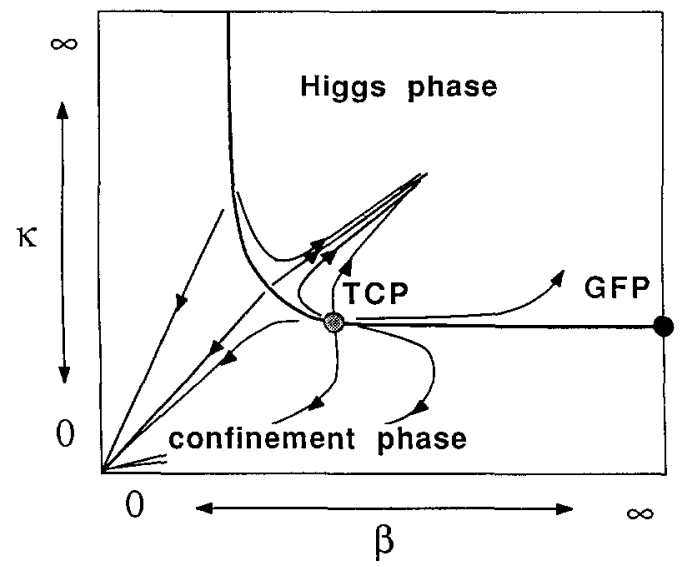

Fig. 6. Schematic diagram of the observed $\mathrm{RG}$ flow in the $(\beta, \kappa)$ plane; the PT is indicated by the thick line.

theories, respectively. One may expect a connection between the spike ending at ( $\left.\beta=1.48, \beta_{A}=0.9\right)$ for $\mathrm{SU}(2)$ [31] and the confinement-Higgs tricritical line of the adjoint Higgs model. Schematically the phase diagram compatible with the measured flow structure of our MCRG analysis is illustrated in fig. 7. The PTs separate the Higgs from the confinement phase. The boundary surfaces contain the SU(2) gauge theory (bottom plane), the U(1) gauge theory (top plane) and the $\mathrm{O}(3)$ scalar

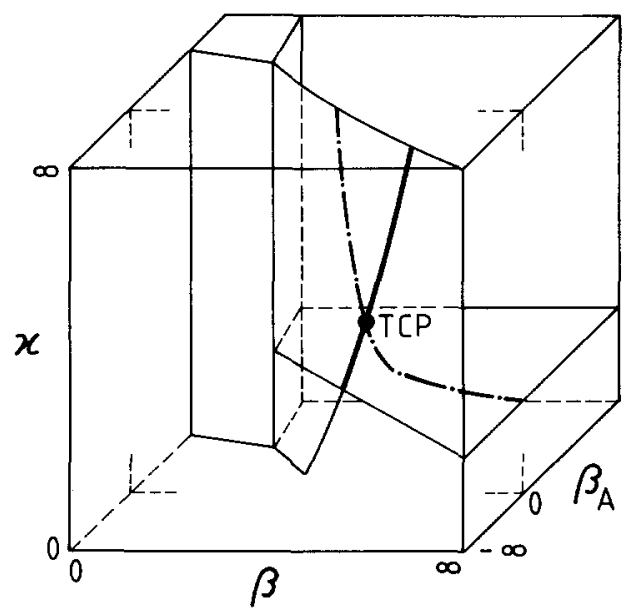

Fig. 7. Expected phase structure in the $\left(0 \leq \beta \leq \infty, 0 \leq \kappa \leq \infty,-\infty \leq \beta_{A}<\infty\right)$ space with the PT surfaces. The thick curve indicates the tricritical line, the dash-dotted one represents the PT in the $\beta_{A}=0$ plane (cf. fig. 6). 
theory (right plane). For comparison with fig. 6 the PT line in the $\beta_{A}=0$ plane is also plotted (dash-dotted curve). In fig. 7 it is assumed that there exists a TCP for $\mathrm{U}(1)$ at finite $\beta_{A}$ [11], although it is not yet excluded that the first order line ends at $\beta_{A}=-\infty$ [22]. However, this possibility is not changing the tricritical structure of the adjoint Higgs model.

Finally let us consider the possible extension of the phase diagram when taking into account finite values of the bare $\Phi^{4}$ coupling $\lambda$. The TCP candidate observed by us in the $\lambda=\infty$ case lies on a tricritical line in the PT surface $\kappa=\kappa_{\mathrm{PT}}(\beta, \lambda)$ at the boundary between a first order (smaller $\beta$ ) and a second order (larger $\beta$ ) part. We expect that for decreasing $\lambda$ the TCP moves towards larger $\beta$; it is possible that this line of TCPs even ends at the gaussian FP at $\beta=\infty, \lambda=0, \kappa=\frac{1}{4}$. For the abelian Higgs model the existence of such a tricritical line was conjectured recently by Nill [37], but this suggestion seems to be valid also for the non-abelian case. Then the TCP may be accessible to perturbation theory at small $\lambda$. The tricritical FP may be anywhere on this line and the continuum theory obtained within the tricritical manifold has one additional relevant parameter and may be therefore more restricted than the trivial continuum theory obtained at the gaussian fixed point.

How should one proceed to support or reject the conjectured FP structure? Additional and more precise bulk measurements at sufficiently large $\beta \simeq 3.0$ on sufficiently large lattices $\left(\geq 16^{4}\right)$ may be useful to find out about a two-state signal - if existent. MCRG studies on larger lattices including more couplings may be helpful. Especially, the inclusion of the radial mode might allow to find out about the possible continuation of the tricritical line to the gaussian FP.

We conclude that the MCRG study of the adjoint SU(2) lattice Higgs model reveals a remarkable structure of the phase diagram, which is much harder to obtain by bulk measurements. Nevertheless more work, including analytical one, is required for a complete determination of all the continuum properties of the model under investigation.

Discussions with T. Neuhaus and F. Nill are gratefully appreciated. We acknowledge the support of Rechenzentrum der Ruhr-Universität Bochum and especially of HLRZ at KFA Jülich where the necessary computations were performed.

\section{Appendix}

In this appendix we give the four Schwinger-Dyson equations, from which the couplings $\beta, \kappa$ and $\beta_{A}$ are derived. Altogether 26 different expectation values (fig. 2) 
414

R. Baiter et al. / Lattice Highs model

have to be measured. The equations are

$$
\begin{aligned}
& 2\left\langle\operatorname{Tr}\left[L(y, \rho, y+\rho) U_{y, \rho}^{\dagger}\right]\right\rangle \\
& =-\kappa \sum_{[\mu]} \frac{1}{2}\left\langle\operatorname{Tr}\left[L(y, \rho, y+\rho) U_{y, \rho}^{\dagger}\right] \operatorname{Tr}\left[L(y, \mu, y+\mu) U_{y, \mu}^{\dagger}\right]\right\rangle \\
& -\kappa \sum_{[\mu]}\left\langle\operatorname{Tr}\left[U_{y, \rho} \Phi_{y+\rho}^{\dagger} U_{y, \rho}^{\dagger} U_{y, \mu} \Phi_{y+\mu}^{\dagger} U_{y, \mu}^{\dagger}\right]\right\rangle, \\
& \left\langle\operatorname{Tr}\left[L(y, \rho, y+\rho) U_{y, \rho}^{\dagger}\right]\right\rangle \\
& =\kappa-\frac{1}{4} \kappa\left\langle\operatorname{Tr}^{2}\left[L(y, \rho, y+\rho) U_{y, \rho}^{\dagger}\right]\right\rangle \\
& +\beta \sum_{(\mu)} \frac{1}{4}\langle\operatorname{Tr}[L(y, \rho, y+\rho) G(y, \rho, \mu)]\rangle \\
& -\beta \sum_{(\mu)} \frac{1}{8}\left\langle\operatorname{Tr}\left[L(y, \rho, y+\rho) U_{y, \rho}^{\dagger}\right] \operatorname{Tr}\left[U_{y, \rho} G(y, \rho, \mu)\right]\right\rangle \\
& +\beta_{A} \sum_{(\mu)} \frac{1}{3}\left\langle\operatorname{Tr}[L(y, \rho, y+\rho) G(y, \rho, \mu)] \operatorname{Tr}\left[U_{y, \rho} G(y, \rho, \mu)\right]\right\rangle \\
& -\beta_{A} \sum_{(\mu)} \frac{1}{6}\left\langle\operatorname{Tr}\left[L(y, \rho, y+\rho) U_{y, \rho}^{\dagger}\right] \operatorname{Tr}^{2}\left[U_{y, \rho} G(y, \rho, \mu)\right]\right\rangle, \\
& 3\left\langle\operatorname{Tr}\left[U_{y, \rho} G(y, \rho, \mu)\right]\right\rangle \\
& =2 \kappa\langle\operatorname{Tr}[L(y, \rho, y+\rho) G(y, \rho, \mu)]\rangle \\
& -\kappa\left\langle\operatorname{Tr}\left[U_{y, \rho} G(y, \rho, \mu)\right] \operatorname{Tr}\left[L(y, \rho, y+\rho) U_{y, \rho}^{\dagger}\right]\right\rangle \\
& +\beta \sum_{(\nu)}\left\langle\operatorname{Tr}\left[G(y, \rho, \nu)^{\dagger} G(y, \rho, \mu)\right]\right\rangle \\
& -\beta \sum_{(\nu)} \frac{1}{2}\left\langle\operatorname{Tr}\left[U_{y, \rho} G(y, \rho, \mu)\right] \operatorname{Tr}\left[U_{y, \rho} G(y, \rho, \nu)\right]\right\rangle \\
& +\beta_{A} \sum_{(v)} \frac{4}{3}\left\langle\operatorname{Tr}\left[G(y, \rho, \nu)^{\dagger} G(y, \rho, \mu)\right] \operatorname{Tr}\left[U_{y, \rho} G(y, \rho, \nu)\right]\right\rangle \\
& -\beta_{A} \sum_{(v)} \frac{2}{3}\left\langle\operatorname{Tr}\left[U_{y, \rho} G(y, \rho, \mu)\right] \operatorname{Tr}^{2}\left[U_{y, \rho} G(y, \rho, \nu)\right]\right\rangle,
\end{aligned}
$$




$$
\begin{aligned}
\left\langle\operatorname{Tr}^{2}[\right. & \left.\left.U_{y, \rho} G(y, \rho, \mu)\right]\right\rangle-1 \\
= & \kappa_{2}^{\frac{1}{2}}\left\langle\operatorname{Tr}[L(y, \rho, y+\rho) G(y, \rho, \mu)] \operatorname{Tr}\left[U_{y, \rho} G(y, \rho, \mu)\right]\right\rangle \\
& -\kappa_{\frac{1}{4}}\left\langle\operatorname{Tr}^{2}\left[U_{y, \rho} G(y, \rho, \mu)\right] \operatorname{Tr}\left[L(y, \rho, y+\rho) U_{y, \rho}^{\dagger}\right]\right\rangle \\
& +\beta \sum_{(\nu)} \frac{1}{4}\left\langle\operatorname{Tr}\left[U_{y, \rho} G(y, \rho, \nu)\right] \operatorname{Tr}\left[G(y, \rho, \nu)^{\dagger} G(y, \rho, \mu)\right]\right\rangle \\
& -\beta \sum_{(\nu)} \frac{1}{8}\left\langle\operatorname{Tr}^{2}\left[U_{y, \rho} G(y, \rho, \mu)\right] \operatorname{Tr}\left[U_{y, \rho} G(y, \rho, \nu)\right]\right\rangle \\
& +\beta_{A} \sum_{(\nu)} \frac{1}{3}\left\langle\operatorname{Tr}\left[G(y, \rho, \nu)^{\dagger} G(y, \rho, \mu)\right] \operatorname{Tr}\left[U_{y, \rho} G(y, \rho, \nu)\right]\right. \\
& \left.\quad \times \operatorname{Tr}\left[U_{y, \rho} G(y, \rho, \mu)\right]\right\rangle \\
& -\beta_{A} \sum_{(\nu)} \frac{1}{6}\left\langle\operatorname{Tr}^{2}\left[U_{y, \rho} G(y, \rho, \mu)\right] \operatorname{Tr}^{2}\left[U_{y, \rho} G(y, \rho, \nu)\right]\right\rangle .
\end{aligned}
$$

The following abbreviations are used

$$
\begin{aligned}
\sum_{[\mu]} & =\sum_{\mu>0}+\sum_{\mu<0}, \\
\sum_{(\nu)} & =\sum_{\nu>0, \nu \neq \rho}+\sum_{\nu<0, \nu \neq-\rho}, \\
G(x, \mu, \nu) & =U_{x+\mu, \nu} U_{x+\nu, \mu}^{\dagger} U_{x, \nu}^{\dagger}, \\
L(y, \rho, x) & =\Phi_{y} U_{y, \rho} \Phi_{x}^{\dagger} .
\end{aligned}
$$

In order to increase the statistics the averages appearing in the above SchwingerDyson equations are taken over the whole lattice.

\section{References}

[1] H. Georgi and S.L. Glashow, Phys. Rev. Lett. 28 (1972) 1494

[2] J. Jersák, in Lattice gauge theories - A challenge in large scale computing, Wuppertal (1985), eds B. Bunk et al. (Plenum, New York, 1986);

R.E. Shrock, Nucl. Phys. B (Proc. Suppl.) 4 (1988) 373

[3] C.B. Lang, C. Rebbi, and M. Virasoro, Phys. Lett. 104B (1981) 294;

R. Brower, D. Kessler, T. Schalk, H. Levine and M. Nauenberg, Phys. Rev. D25 (1982) 3319;

F. Karsch, E. Seiler and I.O. Stamatescu, Phys. Lett. 131B (1983) 138

[4] R. Baier, R.V. Gavai and C.B. Lang, Phys. Lett. 172B (1986) 387

[5] I.-H. Lee and J. Shigemitsu, Nucl. Phys. B263 (1986) 280;

G. Schierholz, J. Seixa and M. Teper, Phys. Lett. 151B (1985) 69; 157B (1985) 209 
[6] A.M. Horowitz, Nucl. Phys. B235 [FS11] (1984) 563

[7] R. Baier and H.-J. Reusch, Nucl. Phys. B285 [FS19] (1987) 535

[8] R. Dashen and H. Neuberger, Phys. Rev. Lett. 50 (1983) 1897;

P. Hasenfratz and J. Nager, Z. Phys. C37 (1988) 477; further references are found in ref. [2]

[9] A. Hasenfratz and P. Hasenfratz, Phys. Rev. D34 (1986) 3160;

I. Montvay, Nucl. Phys. B293 (1987) 479

[10] D.J.E. Callaway and R. Petronzio, Nucl. Phys. B267 (1986) 253

[11] J. Jersák, T. Neuhaus and P. Zerwas, Phys. Lett. 133B (1983) 103;

H.G. Evertz, J. Jersák, T. Neuhaus and P. Zerwas, Nucl. Phys. B251 [FS13] (1985) 279

[12] S.K. Ma, Phys. Rev. Lett. 37 (1976) 461;

R.H. Swendsen, Phys. Rev. Lett. 42 (1979) 859;

R.H. Swendsen, in Real-space renormalization, ed. by T.W. Burkhardt and J.M.J. van Leeuwen, Topics in current physics, vol. 30 (Springer-Verlag, Berlin, 1982)

[13] R.B. Griffiths, Physica 106A (1981) 59

[14] D.P. Landau and R.H. Swendsen, Phys. Rev. Lett. 46 (1981) 1437; Phys. Rev. B33 (1986) 7700

[15] B. Nienhuis and M. Nauenberg, Phys. Rev. Lett. 35 (1975) 477;

M.E. Fisher and A.N. Berker, Phys. Rev. B26 (1982) 2507

[16] A. Hasenfratz and P. Hasenfratz, Nucl. Phys. B295 [FS21] (1988) 1;

P. Hasenfratz, in Computational Physics, ed. R.D. Kenway and G.S. Pawley (Scottish Univ. Press, 1987)

[17] R. Gupta, M.A. Novotny and R. Cordery, Phys. Lett. 172B (1986) 86

[18] A.N. Burkitt, Nucl. Phys. B270 [FS16] (1986) 575

[19] C.B. Lang, Phys. Rev. Lett. 57 (1986) 1828, Nucl. Phys. B280 [FS18] (1987) 255

[20] C.B. Lang and C. Rebbi, Phys. Rev. D35 (1987) 2510

[21] K. Decker, A. Hasenfratz and P. Hasenfratz, Nucl. Phys. B295 [FS21] (1988) 21

[22] A. Hasenfratz, Phys. Lett. 201B (1988) 492

[23] A. Gonzalez-Arroyo, M. Okawa and Y. Shimizu, Phys. Rev. Lett. 60 (1988) 487

[24] R. Baier, C.B. Lang and H.-J. Reusch, Nucl. Phys. B (Proc. Suppl.) 4 (1988) 407

[25] K.G. Wilson, Phys. Rev. D10 (1974) 2445

[26] G.J. Daniell, A.J.G. Hey and J.E. Mandula, Phys. Rev. D30 (1984) 2230

[27] R.H. Swendsen, Phys, Rev. Lett. 47 (1981) 1775

[28] D.J.E. Callaway, R.C. Furlong and R. Petronzio, Phys. Rev. D35 (1987) 4031

[29] C.B. Lang and M. Salmhofer, Phys. Lett. 205B (1988) 329

[30] K.M. Bitar, Phys. Rev. D34 (1986) 2462

[31] G. Bhanot and M. Creutz, Phys. Rev. D24 (1981) 3212

[32] K.G. Wilson, in Recent developments of gauge theories, ed. G. 't Hooft et al. (Plenum, New York, 1982);

R.H. Swendsen, in Phase transitions, ed. M. Levy, J.C. Le Guillou and J. Zinn-Justin (Plenum, New York, 1982);

P. Hasenfratz, Erice Lectures, 1984, preprint CERN TH-3999/84

[33] R.H. Swendsen, Phys. Rev. Lett. 52 (1984) 1165

[34] M. Falcioni, G. Martinelli, M.L. Paciello, G. Parisi and B. Taglienti, Nucl. Phys. B265 [FS15] (1986) 187 ;

A. González-Arroyo and M. Okawa, Phys. Rev. D35 (1987) 672;

D.J.E. Callaway and R. Petronzio, Nucl. Phys. B292 (1987) 497

[35] H.W. Blöte and R.H. Swendsen, Phys. Rev. Lett. 43 (1979) 799

[36] V. Grösch et al., Phys. Lett. 162B (1985) 171

[37] F. Nill, thesis, Universität München (1987), unpublished; private communication 